\title{
Managing Cost Uncertainty in Production Using Genetic Algorithm
}

\author{
Dr. Niju. P. Joseph ${ }^{1}$, Redha Jasim Shaker ${ }^{2}$
}

\section{Introduction}

Efficient and effective management of production cost is carried out by identifying the uncertainty in production planning in supply chain. Thus the determination of the uncertainty factors at various levels in a supply chain becomes inevitable so as to ensure minimal Cost for the manufacturing process. Minimizing the total supply chain cost is meant for minimizing labour, material and minimizing cost in the entire supply chain.

The minimization of the total production cost can only be achieved when is carried out at each member of the production chain. For production planning, one typically needs to determine the variable production costs, including manufacturing costs, Labor cost, materials cost, inventory holding costs, and any relevant resource acquisition costs. A serious issue in the implementation of the same is that cost level is not static for every period and for every category. In this paper, we have developed a new and efficient approach that works on Genetic Algorithms in order to minimize production cost.

The competitiveness of a company in the modern-day market place is determined by more than one vital feature such as the decrease in lead times and expenses, enhancement of customer service levels and upgrading the product quality. The business organizations have started to ponder over the supply chains due to the aforesaid factors. A supply chain can be defined as a collection of companies offering products and services to the market. A supply chain can be illustrated as an incorporation of multiple entities that work in coalition towards

a) Obtaining raw materials.

b) Converting these raw materials into precise end products.

c) Delivering the end products to retailers.

Supply chain management involves a set of procedures that aid in the proficient integration of suppliers, manufacturers, warehouses and stores to ensure appropriate production and distribution of right quantities to the right location on right time and reducing the total supply chain cost as a result in addition to fulfilling service level requirements. The manufacturer, who acquires the raw materials, converts them into end products and distributes the same to the customers, is regarded as the manager of the supply chain.

The management of the dynamic demand is a huge process that numerous supply chain firms indented towards decreasing the supply chain costs besides enhancing customer service levels face. The concepts of supply chain management incorporates a wide range of activities that support the planning, implementation and control manufacturing and the delivery processes right from the source of raw material to the spot where the end product is utilized .

Acute issues in supply chain management arise out of shorter product Lifecycles that lead to higher demand uncertainty and the action on global markets consequently increasing the supply chain complexity.

Uncertainty relates to the situation in which there is a total absence of information or awareness of a potential event occurrence, irrespective of whether the outcome is positive or negative. The term risk and uncertainty are frequently used interchangeably as firms move to leaner operating models and increasingly leverage global sourcing models, uncertainty in both supply and demand is growing along with supply chain complexity.

To improve the overall performance of supply chain, the members of supply chain may behave as a part of a unified system and coordinate with each other. Thus "coordination" comes into focus.

\section{Categories of Uncertainty}

Uncertainty can be measured by the frequency of its occurrence, and analyzing the relative contribution and resulting effect on delivery performance can quantify whether the impact is minor or major. Eight uncertainties that are most likely to affect customer delivery performance are external late supply, internal late supply, planned set-up time exceeded, machine breakdowns, labor unavailability, tooling unavailability, demand batch size enlargement and customer design changes.

Their simulation output highlighted four uncertainties that have significant effects to PDL (parts delivered late) and FPDL (finished product delivered late). These are external late supply, machine break-downs, demand batch size enlargement and customer design changes.

The concept of 'yield factor' is used to embrace system uncertainties. A composed yield factor relates the quantities of required inputs to satisfy a demand of specified output when the system uncertainties cause losses of articles in different levels of the production process.

Therefore, the composed yield factor is a function of the prominent production factors in the different stages of the process.

\section{Evaluating Uncertainty}

Uncertainty can be measured by the frequency of its occurrence, and analyzing the relative contribution and

\section{Volume 6 Issue 7, July 2017




\section{International Journal of Science and Research (IJSR) \\ ISSN (Online): 2319-7064}

Index Copernicus Value (2015): 78.96 | Impact Factor (2015): 6.391

resulting effect on delivery performance. It can quantify whether the impact is minor or major. Many conceptual and mathematical models are proposed and used to manage competitive production/manufacturing Uncertainties in manufacturing have heterogeneous effects due to the interrelationships between resources and operations.

The lead-time and demand uncertainties are individually and interactively significant determinants of system performance found that as processing time uncertainty increases, simple dispatch heuristics provide performance comparable or superior to that of algorithmically more sophisticated scheduling policies. Again, increasing manufacturing flexibility leads to increased performance and to knob the uncertainty.

\section{Building GA}

In building a Genetic Algorithm GA methodology to solve the supply chain sourcing problem, six fundamental issues that affect the performance of the GA must be addressed. They are chromosome representation, initialization of the population, selection strategy, genetic operators, termination criteria, and evaluation measures. In the following subsections, these issues are introduced and described specifically for the proposed multi-objective GA.

\subsection{Chromosome Representation}

For any GA, a chromosome representation is needed to describe each individual in the population. Chromosome representation determines how the problem is structured in the GA, as well as the genetic operators that can be used. For the sourcing decision, the chromosome representation in this case is fairly straightforward. Notice, that not all combinations of the decision variables constitute a feasible solution.

\subsection{Initialization of the Population}

The initial population is formed randomly based on the upper and lower bound for each of the decision variables in a chromosome using a uniform distribution.

\subsection{Selection Strategy}

Selection of parents to produce successive generations is very important in driving the search. The goal is to give more chance to the "fittest" individuals to be selected. For each selection scheme, probabilities are assigned to the individuals. The better individuals have higher probabilities.

Normalized individuals are first ranked from best to worst according to their fitness values. Then each individual is assigned a probability based on the rank from a truncated geometric distribution. In the original NSGA-II method, a tournament selection is used. Since the population is sorted from best to worst, the normalized geometric ranking scheme does not require any more sampling or sorting as does the tournament selection.

\subsection{Genetic Operators}

Reproduction is carried out by the application of genetic operators on selected parents. Four mutation (Boundary, Uniform, No uniform, and Multi-No Uniform) and three crossover operators (Simple, Arithmetic, and Heuristic) are used based on the representation. Continuous variables use the version by Michalewicz (1996) while the discrete variables use the modifications by Joines et al. (1996).

\subsection{Termination Criteria}

The GA is terminated after a specified number of generations.

\subsection{Single Point Crossover}

A single crossover point on both parents' organism strings is selected. All data beyond that point in either organism string is swapped between the two parent organisms. The resulting organisms are the children.

\subsection{Uniform Mutation}

A mutation operator that replaces the value of the chosen gene with a uniform random value selected between the user-specified upper and lower bounds for that gene. This mutation operator can only be used for integer and float genes.

\subsection{Tournament selection}

It is a method of selecting an individual from a population of individuals in a genetic algorithm. Tournament selection involves running several "tournaments" among a few individuals chosen at random from the population. The winner of each tournament (the one with the best fitness) is selected for crossover. Selection pressure is easily adjusted by changing the tournament size. If the tournament size is larger, weak individuals have a smaller chance to be selected.

Tournament selection pseudo code:

Choose $K$ (the tournament size) individuals from the population at random

Choose the best individual from pool/tournament with probability $\mathrm{p}$ Choose the second best individual with probability $\mathrm{p}^{*}(1-\mathrm{p})$ Choose the third best individual with probability $\mathrm{p}^{*}\left((1-\mathrm{p})^{\wedge} 2\right)$.

Deterministic tournament selection selects the best individual (when $\mathrm{p}=1$ ) in any tournament. A 1-way tournament $(\mathrm{k}=1)$ selection is equivalent to random selection. The chosen individual can be removed from the population that the selection is made from if desired, otherwise individuals can be selected more than once for the next generation.

Tournament selection has several benefits: it is efficient to code, works on parallel architectures and allows the selection pressure to be easily adjusted. 


\section{International Journal of Science and Research (IJSR) \\ ISSN (Online): 2319-7064}

Index Copernicus Value (2015): 78.96 | Impact Factor (2015): 6.391

\subsection{Evaluation Measure}

Genetic algorithms rely on the simple premise of using natural selection as a means of problem solution. The objective function is the driving the force of the GA search. In this research, instead of performing an analytical function evaluation, each solution is simulated to determine its performance. Because the simulation is based on a particular forecasted demand level and the answers generated need to be as robust as possible.

\section{Fitness Function}

Fitness functions ensure that the evolution is toward optimization by calculating the fitness value for each individual in the population. The fitness value evaluates the performance of each individual in the population.

The fitness function is given by:

\section{$F(x)=\log (1-X c / X p), k=1,2,3 . . m$}

Where Xc- count of Occurrences in the dataset and Xp-the total values in the dataset

Total Manufacturing cost

$\mathrm{TMC}=$ Purchasing Cost $(\mathrm{PC})+$ Operating Cost $(\mathrm{OC})+$ Storage

$\operatorname{Cost}(\mathrm{SC})$. (1)

Objective Function is minimizes the total cost:

Minimize $\Sigma$ Total Manufacturing Cost

$\mathrm{PC}=\Sigma \mathrm{Q} \times \mathrm{P}$

$\mathrm{OC}=\mathrm{N} \times \mathrm{Co} \ldots \ldots . .(4)$

$\mathrm{SC}=\Sigma \mathrm{SQt} \times \mathrm{Cs} \ldots \ldots(5)$

- $\quad \mathrm{TMC}=$ Total Manufacturing Cost

- $\mathrm{PC}=$ Purchasing Cost

- $\mathrm{OC}=$ Operating Cost

- $\mathrm{SC}=$ Storage Cost

- $\mathrm{Q}=$ Quantity of material

- $\mathrm{P}=$ unit price of material

- $\mathrm{N}=$ No. of Units made

- $\mathrm{Co}=$ average production cost including Labor cost for making a single Unit.

- $\mathrm{Cs}=$ storage cost

- $\mathrm{SQt}=$ Stock Quantity at time

\subsection{Chromosome Representation}

The Uncertain Variables are constructed in the format of a chromosome.

\begin{tabular}{|l|l|l|l|l|l|}
\hline Qi & Pi & N & Co & Sqt & Cs \\
\hline
\end{tabular}

Single point Crossover Uniform Mutation Genetic Algorithm (SCUMGA)

The Proposed work considers the uncertainty of cost in production scenario. It develops an algorithm for managing uncertainty by minimizing manufacturing cost by crossover $\&$ mutation operators.

Step1: Assign values for population size, maximum generation, initial crossover probabilities, initial mutation probabilities, weight values, number of objectives, and number of variable.

Step 2: Generate initial population $P$ based on population size. Step 3: Compute the fitness value of each individual in $P$.

Step 3: If stopping criterion is satisfied then the algorithm will break. Step 5: Combine $n$ objective function into single function using weight values.

Step 4: In the tournament selection [3], two individuals are selected at random and the fittest is selected into mating. Mating pool will keep the selected individuals for reproduction.

Step 5: Perform crossover and mutation. Use the single point crossover [3] to randomly select cross points and swap between individuals. Uniform mutation [3] randomly select mutate points and replace it with randomly generated value.

Step 6: The best individuals will be included in the new population for the next generation. This is to ensure that the best individuals make it into the next generation. Increment generation counter (gen+1) and go to step 3 .

\section{Discussion and Results}

SCUMGA Algorithm is applied to a production control system in a supply chain. The Uncertainty regarding the production cost will affect the profit margins of any business entity. In a production system, there are various factors that play a significant role. The input data is collected from normalized data of UK based ASTEL computer production dataset [Table 6.1].

The two initial chromosomes are generated at the beginning of the genetic algorithm. These initial Chromosomes are subjected for the genetic operators, Crossover and Mutation. The resultant chromosome thus obtained again processed with repeat crossover and mutation that it moves towards the best chromosome after the each iterative execution. Hence at the end of the execution of 100 iterations, best chromosome ' $9,31,5,50,70,10$ ' is obtained. This generates an optimal TMC value of 1229 .

Table 6.1:.Manufacturing Cost Dataset A

\begin{tabular}{|c|c|c|c|c|c|c|c|}
\hline Prod id & Qi & Pi & N & C o & Sqt & Cs & TMC \\
\hline 1 & 12 & 31 & 5 & 50 & 70 & 10 & 1794 \\
\hline 2 & 14 & 35 & 9 & 60 & 80 & 13 & 2070 \\
\hline 3 & 11 & 36 & 8 & 77 & 92 & 14 & 2300 \\
\hline 4 & 9 & 38 & 13 & 61 & 76 & 12 & 2047 \\
\hline 5 & 17 & 45 & 12 & 55 & 69 & 15 & 2460 \\
\hline 6 & 14 & 46 & 11 & 61 & 78 & 16 & 2563 \\
\hline 7 & 15 & 41 & 12 & 55 & 81 & 14 & 2409 \\
\hline 8 & 13 & 40 & 8 & 62 & 99 & 17 & 2699 \\
\hline
\end{tabular}

While applying this genetic algorithm with the past records, it can be understood that controlling this resultant chromosome is sufficient to reduce the total operating cost by due to the fluctuating production or labor or machine cost. The Genetic Algorithm optimizes the total operating cost by optimizing the production variables like manufacturing, labor and machine cost. 
International Journal of Science and Research (IJSR)

ISSN (Online): 2319-7064

Index Copernicus Value (2015): 78.96 | Impact Factor (2015): 6.391
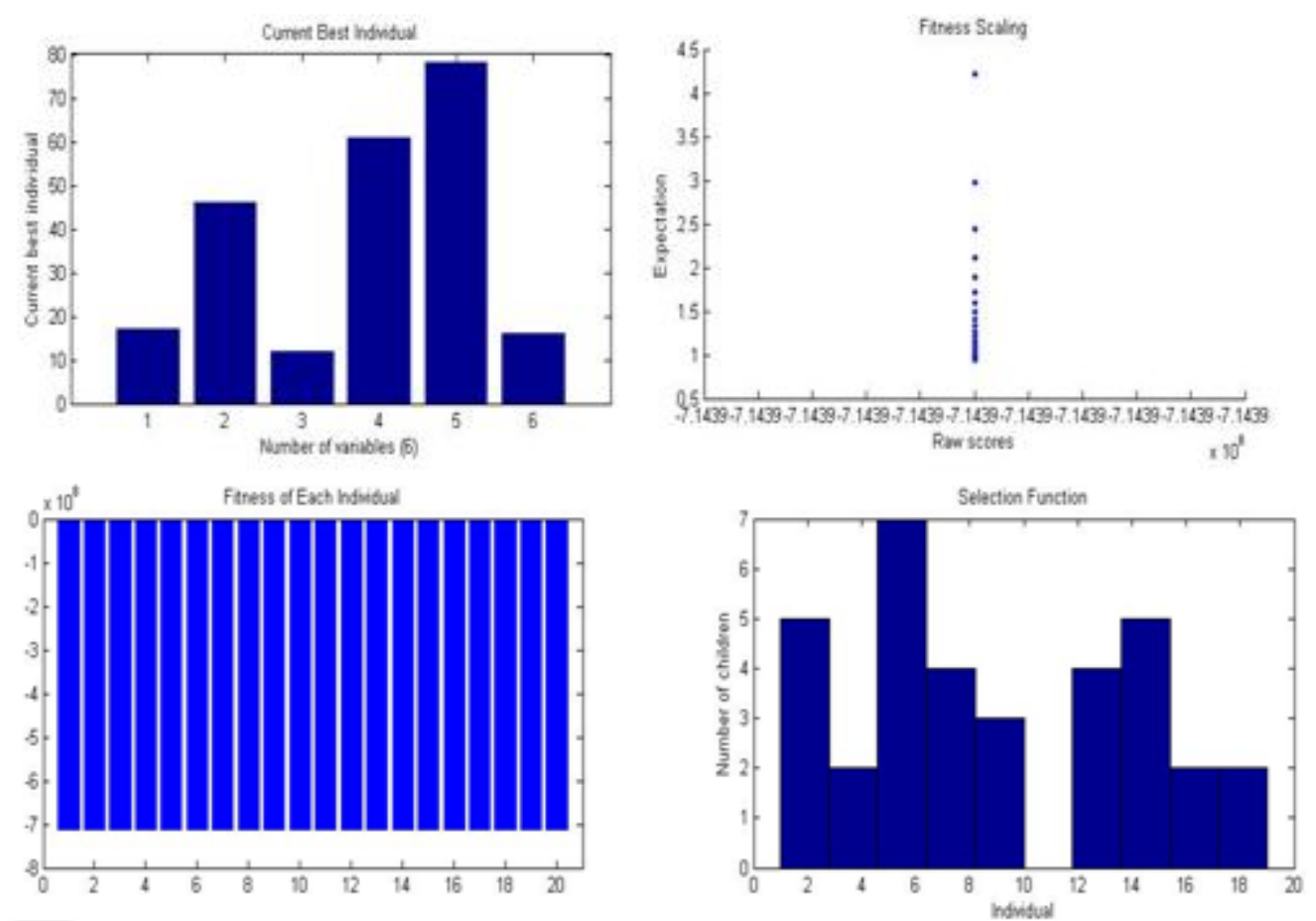

Figure 6.1: Result obtained by GA tool implementation

The SCUMGA algorithm is compared with standard GA's like NSGA-I and NSGA-II and found to perform better. The SCUMGA algorithm performs $13.4 \%$ better than NSGA-I and $18.6 \%$ than NSGA-II (Table 6.2).

Table 6.2: Comparison of the performance of Algorithms

\begin{tabular}{|c|c|}
\hline Algorithm & $\begin{array}{c}\text { TMC (Total Manufacturing } \\
\text { Cost) }\end{array}$ \\
\hline NSGA-I & 1420 \\
\hline NSGA-II & 1510 \\
\hline SCUMGA & 1229 \\
\hline
\end{tabular}

\section{Conclusion}

The Algorithm uses multiple crossover and mutation operators for managing removing uncertainty in production cost evaluation. This can lead to better forecasting of production cost and thereby achieve higher performance levels.MATLAB is used to obtain the best solution. The Uncertain variables can be controlled and hence the production cost can be reduced leading to lower supply chain cost.

\section{References}

[1] Kaur Arshinder, Arun Kanda, and S.G. Deshmukh ,2008,"A Review on Supply Chain Coordination: Coordination Mechanisms, Managing Uncertainty and Research Directions".Springer-Verlag.

[2] Miguel Zamarripa, Javier Silvente and Antonio Espuña, 2012,"Supply Chain Planning under uncertainty using Genetic Algorithms" .J.Computer Aided Chemical Engineering, Vol.30, pp.457-461

[3] Chandrasekaran Sowmya Danalakshmi, Gabriel Mohan Kumar,2008."'Optimization of Supply Chain Network Using Genetic Algorithm”,
[4] Zheng yahong,2012," Supply Chain Management under availability \& uncertainty", Doctoral Thesis submitted to Laboratoire d'Automatique, Genie Informatique et Signal (LAGIS), France.

[5] Patcharee Boonyathan, Damien Power, 2007,',Impact of Supply Chain Uncertainty on Business Performance and the Role of Supplier and Customer Relationships: Comparison between Product and Service Organization",University of Melbourne.

[6] C.N. Verdouw1,2, A.J.M. Beulens2,2011,”Agile Information Systems for Mastering Supply Chain Uncertainty", Handbook- Supply Chain Management New Perspectives.

[7] Yufu Ning, Huanbin Sha, Lixia Rong,2012,'Two-stage Supply Chain Model with Uncertain Demand", Proceedings of the Twelfth International Conference on Electronic Business, Xi'an, China.

[8] Rachel,Denis,1998,"Shrinking the supply chain uncertainty circle", IOM control.

[9] Rachel Mason-Jones, and Denis R. Towill ,2000,"Coping with Uncertainty:Reducing "Bullwhip" Behaviour in Global Supply Chains" Supply Chain Forum An International Journal $\mathrm{N}^{\circ} 1$.

[10] J. Mula!, R. Poler, J.P. Garcia Sabater, F.C. Lario,2006, Models for production planning under uncertainty: A review", International Journal of Production Economics Volume 103, Issue 1, September 2006, Pages 271-285.

[11] Jack G.A.J. van der Vorst, 2002,'”Identifying sources of uncertainty to generate supply chain redesign strategies, International Journal of Physical Distribution \& Logistics Management.

[12] Jorge Casillas a, Francisco J. Mart1'nez-Lo'pez ,2009,"Mining uncertain data with multiobjective genetic fuzzy systems to be applied in consumer behaviour modelling". Expert Syst. Appl. 36(2): 16451659 . 


\section{International Journal of Science and Research (IJSR) \\ ISSN (Online): 2319-7064}

Index Copernicus Value (2015): 78.96 | Impact Factor (2015): 6.391

[13]Luciano Sáncheza, InésCousob, JorgeCasillas,2009,"'Genetic learning of fuzzy rules based on low quality data", Fuzzy Sets and Systems 160(17): 2524-2552 .

[14]Lawrence V. Snyder ,2006,"Supply and Demand Uncertainty in Multi-Echelon Supply Chains", Lehigh University.

[15]Martin Christopher,2012,"Managing Supply Chain Complexity in an Age of Uncertainty", Lecture Notes,Cranfield University,U.K.

[16] Fatemeh Forouzanfar and Reza Tavakkoli-Moghaddam ,2012,'Using a genetic algorithm to optimize the total cost for a location-routing-inventory problem in a supply chain with risk pooling”, Journal of Applied Operational Research.

[17] Kumaraguru Mahadevan,2007,"Supply Chain Uncertainty: An insight for Australian CEO's and Managers". Proceedings of 8th International Research Conference onQuality, Innovation and Knowledge Management.

[18] Jyri Vilko*, Jan Edelmann, Jukka Hallikas,2012,"Defining the levels of uncertainty in supply chains Jyri Vilko*, Jan Edelmann, Jukka Hallikas, Research paper, Lappeenranta University of Technology,Finland. 